Proceedings of the International Symposium on Physics of Materials (ISPMA 14), September 10-15, 2017, Prague

\title{
A Model of Microstructure Evolution in Metals Exposed to Large Strains
}

\author{
J. KRATOCHVÍl ${ }^{a, b, *}$ AND M. KRUŽÍK ${ }^{b, c}$ \\ ${ }^{a}$ Charles University, Faculty of Mathematics and Physics, Sokolovská 83, 18675 Prague, Czech Republic \\ ${ }^{b}$ Czech Technical University, Faculty of Civil Engineering, Department of Physics, \\ Thákurova 7, 16629 Prague, Czech Republic \\ ${ }^{c}$ Academy of Sciences of Czech Republic, Institute of Information Theory and Automation of the ASCR, \\ Pod vodárenskou věží 4, 18208 Prague, Czech Republic
}

\begin{abstract}
Crystalline materials at yield behave as anisotropic, highly viscous fluids. A microscopic inspection reveals a structural adjustment of the crystal lattice to the material flow carried by dislocations. The resistance to this flow determines the strength of ductile materials. The deformation microstructure evolves within a common framework up to very high strains $>100$. To avoid energetically costly multislip, materials are subdivided into regions which deform by fewer slip systems. To maintain compatibility, the regions defined as deformation bands occur in a form of elongated alternately misoriented domains filled with fairly equiaxed dislocation cells. In the proposed continuum mechanics model, the formation of deformation bands of a lamellae type is interpreted as a spontaneous deformation instability caused by an anisotropy of hardening. However, such a model of the bands predicts their extreme orientation and their width tends to zero. This trend is opposed by hardening caused by a bowing stress of dislocations within the cells.
\end{abstract}

DOI: 10.12693/APhysPolA.134.753

PACS/topics: 02.70.Bf, 61.72.Lk, 02.30.Jr

\section{Introduction}

The process of the deformation microstructure evolution may be understood as an energetically optimal adjustment of the crystal lattice to the material flow. The evolution is a result of a complex and well documented process reviewed e.g. in [1-4]. It follows a common scenario represented by a succession of the deformation bands in the form of cell blocks, microbands, and finally a laminar structure which dominates the high strain range. In each evolution stage, a new cell wall system ${ }^{\dagger}$ is built up to facilitate the flow by the storage and the annihilation of the excess dislocations and their debris. The maximum achievable deformation strengthening is determined by the resistance to a dislocation flow through the laminar structure.

The laminar structure occurs in materials exposed to e.g. severe rolling, high pressure torsion, accumulative roll bonding or sliding in a wide range of strain levels of order 1 up to over 100 [6, 7]; the proposed model simulates rolling. The formed lamellae are oriented close to the rolling direction, with a large misorientation across their boundaries, $>15^{\circ}$. The lamellae most often contain one cell in the width and several to a few along their length. The lamellae boundaries are frequently lay-

*corresponding author; e-mail: kratochvil@fsv.cvut.cz

${ }^{\dagger}$ In non-cell forming materials the inner structure of the deformation bands consists of dislocations organized into a Taylor-type lattice [5]. ered closely to each other with narrow cells between them forming very thin cell blocks. The narrow channels between the lamellae boundaries are bridged by interconnected cell boundaries which are approximately perpendicular to the rolling direction and thus form a bamboo structure.

The model proposed in the following section points to two main mechanisms inducing the existence and evolution of the laminar structure: (a) material anisotropy caused by crystalline nature of plasticity, and (b) dislocation cells.

\section{A model of laminar structure}

Models of the deformation bands have been proposed by a number of authors, e.g. [8-13], as commented in our previous papers $[14,15]$. The present paper focuses on the deformation bands in the form of a laminar structure filled with cells. As a base, we employ Biot's model of orthotropic materials [16] where the orthotropy is carried by plane strain symmetric double slip. The model has been analyzed by Harren's et al. [17] and employed in our recent publications on deformation bands [14, 15]. The considered symmetric double-slip compression is a convenient two-dimensional idealization of a cold-rolled polycrystalline grain oriented symmetrically with respect to the normal to the rolling plane (so-called rotated cubic orientation) [18].

We consider an infinitely extended rigid-plastic crystal domain in the coordinate system $(x, y)$ with the symmetry axis placed along the $y$ coordinate axis. The rolling direction is parallel to $x$ axis, the direction 
of compression is parallel to $y$ axis. The angle of the slip planes with respect to the $y$ axis is $\phi= \pm 35^{\circ}$. The incremental deformation representing a deviation from the original symmetric double slip is expressed through an incremental displacement vector $\boldsymbol{u}(x, y)$. The incremental strain $\varepsilon(x, y)$ added to the current homogeneous or homogenized strain $\bar{\varepsilon}$ is defined as $\varepsilon=\left(\nabla \boldsymbol{u}+(\nabla \boldsymbol{u})^{T}\right) / 2$. In the rigid-plastic approximation, the incremental strain $\varepsilon$ is caused by increments of slips $\gamma^{(1)}(x, y)$ and $\gamma^{(2)}(x, y)$ on the two considered slip systems $\boldsymbol{\varepsilon}=\sum_{i=1}^{2} \gamma^{(i)}\left(\boldsymbol{s}^{(i)} \otimes \boldsymbol{m}^{(i)}+\boldsymbol{m}^{(i)} \otimes \boldsymbol{s}^{(i)}\right) / 2$; here $\boldsymbol{s}^{(1)}=(\sin \phi, \cos \phi)$ and $\boldsymbol{s}^{(2)}=(-\sin \phi, \cos \phi)$ represent the slip directions, and $\boldsymbol{m}^{(1)}=(-\cos \phi, \sin \phi)$ and $\boldsymbol{m}^{(2)}=(\cos \phi, \sin \phi)$ are the unit slip plane normals, we obtain

$$
\begin{aligned}
& \varepsilon_{x x}=-\sin 2 \phi\left(\gamma^{(1)}+\gamma^{(2)}\right) / 2, \\
& \varepsilon_{x y}=-\cos 2 \phi\left(\gamma^{(1)}-\gamma^{(2)}\right) / 2 .
\end{aligned}
$$

To satisfy the incompressibility of the deformation $\varepsilon_{x x}+\varepsilon_{y y}=\partial_{x} u_{x}+\partial_{y} u_{y}=0$, we use the stream function $\psi$ so that $u_{x}=\partial_{y} \psi, u_{y}=-\partial_{x} \psi$. We look for an inhomogeneous, kinematically admissible deformation constructed from the stream function $\psi=F(x+\xi y)$ which represents an inhomogeneous simple shear parallel to the planes $x+\xi y=$ constant. The shear inhomogeneity can be interpreted as a deformation band-like pattern perpendicular to the direction $\xi=\tan \theta$, where $\theta$ is the angle between the $x$ axis and the normal to the bands. In view of the linearity of the problem at hand, any linear combination of the stream functions with different profiles $F$ and directions $\xi$ is admissible. In terms of the stream function, the admissible strains read

$$
\varepsilon_{x x}=\xi F^{\prime \prime}, \quad \varepsilon_{x y}=\frac{1}{2}\left(\xi^{2}-1\right) F^{\prime \prime},
$$

where $F^{\prime \prime}$ represents the second derivative with respect to the argument $x+\xi y$. Kinematically admissible crystallographic slip increments then follow from (2.1),

$$
\begin{aligned}
& \gamma^{(1)}=\left(\frac{1-\xi^{2}}{2 \cos 2 \phi}-\frac{\xi}{\sin 2 \phi}\right) F^{\prime \prime}, \\
& \gamma^{(2)}=\left(\frac{\xi^{2}-1}{2 \cos 2 \phi}-\frac{\xi}{\sin 2 \phi}\right) F^{\prime \prime} .
\end{aligned}
$$

The stress in the deforming crystal domain is assumed in the form $\overline{\boldsymbol{\sigma}}+\boldsymbol{\sigma}$ where $\overline{\boldsymbol{\sigma}}$ is the applied homogeneous compression in the $y$ direction and $\boldsymbol{\sigma}(x, y)$ is an (in general) inhomogeneous stress increment. The stress equilibrium, $\operatorname{div} \boldsymbol{\sigma}=0$, leads $\mathrm{to}^{\ddagger}$

$$
\partial_{x} \sigma_{x x}+\partial_{y} \sigma_{x y}=0, \quad \partial_{x} \sigma_{x y}+\partial_{y} \sigma_{y y}=0 .
$$

\footnotetext{
¥We leave out the destabilizing effect of geometrical softening caused by pre-stress $\overline{\boldsymbol{\sigma}}$, since in the deformation banding, the prestress causes a subsidiary effect only [14, 15]
}

The slip increments are induced by the increments in the resolved shear stresses $\tau^{(i)}=\boldsymbol{s}^{(i)} \cdot \boldsymbol{\sigma} \boldsymbol{m}^{(i)}, i=1,2$,

$$
\begin{gathered}
\tau^{(1)}=-\sin 2 \phi\left(\sigma_{x x}-\sigma_{y y}\right) / 2-\cos 2 \phi \sigma_{x y}, \\
\tau^{(2)}=-\sin 2 \phi\left(\sigma_{x x}-\sigma_{y y}\right) / 2+\cos 2 \phi \sigma_{x y} .
\end{gathered}
$$

In the rate-independent approximation the resolved shear stress equals the flow stress in the plastic regime. This is accounted for by the hardening rule which plays a central role in the model.

The proposed rule is assumed to be formed by bulk and boundary parts. For the bulk part

$$
\begin{gathered}
\tau_{V}^{(1)}=\left(h+h_{\text {bow }}\right) \gamma^{(1)}+q h \gamma^{(2)}, \\
\tau_{V}^{(2)}=q h \gamma^{(1)}+\left(h+h_{\text {bow }}\right) \gamma^{(2)} .
\end{gathered}
$$

The entries are the self-hardening coefficient $h$ and the latent (cross) hardening coefficient $q h$. The latent-toactive hardening ration $q$ is a measure of the hardening anisotropy and is one of the key parameters of the model. The reason for adding a hardening term $h_{\text {bow }}$ to the self hardening $h$ is that the dislocation bowing within the cells hinders the active slip. We employ a line tension strengthening in the form $h_{\text {bow }}=G b / L$, where $G$ is the shear modulus and $b$ is the magnitude of the Burgers vector. As the lamellae most often contain one cell in the width, the cell size is identified with the lamella width $L$.

The lamella boundary part of the hardening rule represents a complex problem: at boundaries dislocations can be reflected, absorbed or desorbed and/or transmitted directly. The lamella boundaries are treated in a highly simplified way as interface objects characterized by an isotropic hardening rule

$$
\tau_{B}^{(1)}=\tau_{B}^{(2)}=\tilde{h}\left(\Delta \hat{\gamma}^{(1)}+\Delta \hat{\gamma}^{(2)}\right) .
$$

Equation (2.7) can be interpreted as a part of the flow stress increments needed to penetrate a cluster of the lamella boundaries. The jumps $\Delta \hat{\gamma}^{(i)}, i=1,2$, represent the difference in the slip increments across the boundaries between the neighboring lamellae. The coefficient $\tilde{h}$ is very difficult to specify; it enters the model as a fitting parameter.

\section{Variational approach to laminar structure formation}

The variational formulation shows clearly that, for anisotropic hardening, an inhomogeneous deformation leading to the lamellae formation is energetically more favorable than the homogeneous one.

In the variational formulation, we utilize the principle of virtual displacements, $\int \operatorname{div} \boldsymbol{\sigma} \cdot \delta \boldsymbol{u}=0$, where $\delta \boldsymbol{u}(x, y)$ is an arbitrary virtual displacement field. The principle leads to a weak form of the equilibrium Eq. (2.4):

$$
\int_{\Omega}\left(\sigma_{x x} \delta \varepsilon_{x x}+2 \sigma_{x y} \delta \varepsilon_{x y}+\sigma_{y y} \delta \varepsilon_{y y}\right) \mathrm{d} V=0,
$$

where $\Omega$ is the crystal domain in the $x y$ plane.

Introducing the constitutive relations (2.6) and (2.7) into (3.1), expressing $\varepsilon_{x x}, \varepsilon_{y y}, \varepsilon_{x y}, \gamma^{(1)}$ and $\gamma^{(2)}$ through the stream function $F(x+\xi y)$ using $(2.2),(2.3)$, and in 
view of the symmetry of the hardening (2.6) and (2.7), the equilibrium (3.1) can be expressed as an extreme of the functional $J$ requiring its first variation to be zero, $\delta J=0$,

$$
\begin{aligned}
J= & \frac{1}{2}\left\{4\left[H_{x x}+B_{x x}\right] \xi^{2}\right. \\
& \left.+\left[H_{x y}+B_{x y}\right]\left(\xi^{2}-1\right)^{2}\right\} \int_{\Omega}\left(F^{\prime \prime}\right)^{2} \mathrm{~d} V+4 \tilde{H} \xi^{2} \int_{A}\left(F^{\prime \prime}\right)^{2} \mathrm{~d} A,
\end{aligned}
$$

where $A$ is the area of the lamella boundaries contained in the domain $\Omega$, and

$$
\begin{aligned}
& H_{x x}=\frac{h(1+q)}{2 \sin ^{2} 2 \phi}, \quad H_{x y}=\frac{h(1-q)}{2 \cos ^{2} 2 \phi}, \\
& B_{x x}=\frac{h_{\text {bow }}}{2 \sin ^{2} 2 \phi}, \quad B_{x y}=\frac{2 h_{\text {bow }}}{2 \cos ^{2} 2 \phi}, \quad \tilde{H}=\frac{\tilde{h}}{2 \sin ^{2} 2 \phi} .
\end{aligned}
$$

In Eq. (3.2), we supposed that the increments $\gamma^{(1)}$ and $\gamma^{(2)}$ are uniform in each lamella. The slip is adjusted such that the incremental slips alternate in the direction perpendicular to the lamellae and is averaged to zero, i.e. $\Delta \gamma^{(i)}=2 \gamma^{(i)}$. The width $L>0$ of the bands is taken to be the same for all the lamellae.

The inhomogeneous incremental deformation defined by $F(x+\xi y) \neq 0$ is now assumed to be such a deviation from the homogeneous increment that the corresponding strains vanish on average. A homogeneous increment can be formally represented by $F(x+\xi y)=0$. The functional (3.2) thus now represents an energy difference between the homogeneous and inhomogeneous deformation increments. The inhomogeneous deformation increment is energetically favorable if the functional becomes negative, $J<0$. If a kinematically possible deformation fulfilling this condition exists, the homogeneous deformation is unstable and a pattern is formed. There are two main mechanisms of the laminar structure evolution: (a) material anisotropy represented by $H_{x x}$ and $H_{x y}$, and (b) cells and lamella boundaries represented by $B_{x x}, B_{x y}$ and $\tilde{H}$.

\subsection{Anisotropy}

To exclude the cells and lamella boundaries, we take $B_{x x}=0, B_{x y}=0, \tilde{H}=0$ in (3.2); the energetic function in (3.2) is consequently reduced to $4 H_{x x} \xi^{2}+H_{x y}\left(\xi^{2}-1\right)^{2}$. In view of $(3.3)_{1}$ this function may become negative for $h>0$ and $q>1$, i.e. for a dominant latent hardening. This is demonstrated by the graph of the reduced energy function $\bar{J}_{A}(\xi)=J(\xi) / H_{x x} \int\left(F^{\prime \prime}\right)^{2}$ shown in Fig. 1a,

$$
\bar{J}_{A}(\xi)=2 \xi^{2}+K\left(1-\xi^{2}\right)^{2},
$$

where

$$
K=\frac{H_{x y}}{H_{x x}}=\frac{(1-q)}{2(1+q)} \tan ^{2} 2 \phi .
$$

The graph of $\bar{J}_{A}(\xi)$ was evaluated for $\phi=35^{\circ}$ and $q=1.5$. The negative $\bar{J}_{A}<0$ indicates an energy gain in forming the pattern. The inhomogeneous incremental deformation with $\xi \rightarrow \infty$ is the most energetically favorable. It corresponds to the lamella-like pattern perpendicular to the direction of the compression, i.e. $\theta \rightarrow \pi / 2$ (parallel to the rolling direction).

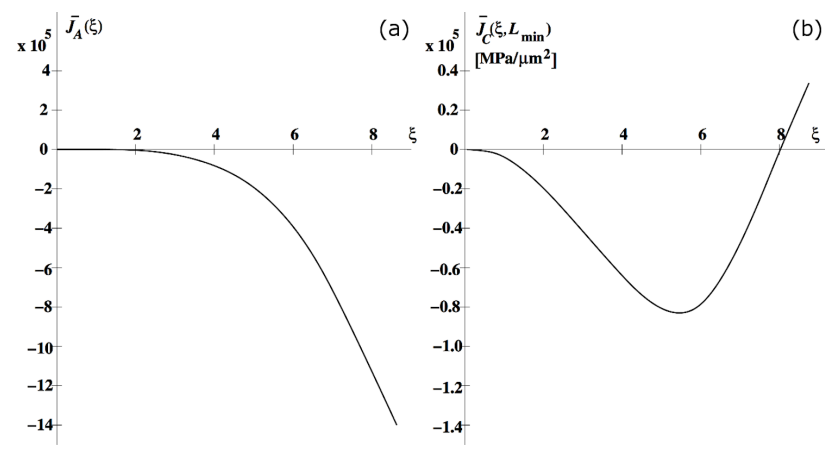

Fig. 1. Graphs of the reduced energetic functions; $\xi$ characterizes the lamellae orientation. (a) $\bar{J}_{A}(\xi)$ indicates a trend to form the lamellae parallel to the rolling direction, $\xi \rightarrow \infty(\theta \rightarrow \pi / 2)$; (b) $\bar{J}_{C}\left(\xi, L_{\min }\right)$ shows a modifying effect of the cells and the lamella boundaries.

To estimate a length scale of the pattern we consider the deformation banding inspired by a band microstructure observed in cell forming metals represented by periodically arranged lamellae perpendicular to the direction $\xi$. The lamellae of the width $L$ are assumed to be homogeneously sheared, i.e. $F^{\prime \prime}= \pm(a / L)$, where $a$ is an amplitude of the incremental displacement. Under these assumptions and $B_{x x}=0, B_{x y}=0$ and $\tilde{H}=0$, the functional (3.2) integrated over the crystal domain $\Omega$ becomes a function

$$
J_{A}(\xi, L)=\frac{1}{2}\left(4 H_{x x} \xi^{2}+H_{x y}\left(\xi^{2}-1\right)^{2}\right) \frac{a^{2}}{L^{2}} V
$$

where $V$ is the volume of the crystal domain $\Omega$. The function (3.6) indicates that for a given amplitude $a$ the fastest growth of the pattern is reached for $L \rightarrow 0$.

That the deformation bands tend to be oriented perpendicularly to the direction of compression and tend to have a zero width was discovered by Biot [16]. He noted ([16] p. 199): "This conclusion may seem paradoxical, but there are inherent limitations in the validity of the theory for very small wavelengths. It is, of course, not valid in the atomic scale. Then non-linearity also enters into play. The result therefore indicates that the buckling wavelength will tend to be the smallest compatible with the small-scale physics of the medium." In the herein proposed model, the small-scale physics is represented by the cells and lamella boundaries.

\subsection{Cells and lamella boundaries}

The functional $J$ (3.2) incorporates the cells and the lamella boundaries through $B_{x x}, B_{x y}$ and $\tilde{H}$. We accept, similarly as in (3.6), the periodically arranged lamellae of the width $L$. In view of that, the area of the lamella boundaries $A$ in the volume $V$ of $\Omega$ is $V / L$. We further assume that the shear strain in the lamellae is $F^{\prime \prime}= \pm(a / L)$; the functional (3.2) integrated over the crystal domain $\Omega$ thus becomes a function

$$
\begin{aligned}
& J_{C}=\frac{1}{2}\left\{4\left[H_{x x}+B_{x x}\right] \xi^{2}+\left[H_{x y}+B_{x y}\right]\left(\xi^{2}-1\right)^{2}\right\} \frac{a^{2}}{L^{2}} V \\
& +\frac{1}{2} 4 \tilde{H} \xi^{2} \frac{a^{2}}{L^{3}} V .
\end{aligned}
$$


Introducing the line tension assumption $h_{\text {bow }}=G b / L$ into (3.7), we obtain for the reduced energetic function

$$
\begin{aligned}
& \bar{J}_{C}(\xi, L)=J_{C}(\xi, L) / H_{x x} \int\left(F^{\prime \prime}\right)^{2}, \\
& \bar{J}_{C}(\xi, L)=\left[2 \xi^{2}+\frac{(1-q)}{2(1+q)}\left(1-\xi^{2}\right)^{2} \tan ^{2} 2 \phi\right] \frac{1}{L^{2}} \\
& +\frac{1}{2 h(1+q)}\left[4 \tilde{h} \xi^{2}+G b\left(1-\xi^{2}\right)^{2} \tan ^{2} 2 \phi\right] \frac{1}{L^{3}} .
\end{aligned}
$$

The minimum of $\bar{J}_{C}(L, \xi)$ with respect to the width $L$ and the orientation $\xi$ indicates the most favorable structure formed. The conditions of the minimum are: $\partial \bar{J}_{C} / \partial L=0$ and $\partial \bar{J}_{C} / \partial\left(\xi^{2}\right)=0$. The graph of $\bar{J}_{C}\left(\xi, L_{\text {min }}\right)$ shown in Fig. 1b demonstrates, in comparison with Fig. 1a, the influence of the inner lamellae structure of the cells and the lamella boundaries.

To demonstrate the predictive ability of the model, an illustrative example of a laminar structure is presented. Due to the drastic simplifying assumptions, the lamella width $L$ and the angle of inclination to the rolling direction $\theta_{\text {roll }}$ predicted by the model are merely indicative. We use entrance data for nickel: $G \approx 70 \mathrm{GPa}$, $b=0.25 \mathrm{~nm}$, i.e. $G b=17 \mathrm{MPa} \mu \mathrm{m}$, and the hardening anisotropy ratio $q=1.5$; the self-hardening $h=500 \mathrm{MPa}$ has been deduced for Ni from the graphs in [3]. The minimization of $\bar{J}_{C}(L, \xi)$ yields: $\xi=5.7$, i.e. $\theta_{\text {roll }} \approx 80^{\circ}$ and $L \approx 0.05 \mu \mathrm{m}$. The fitting parameter $\tilde{h} \approx-0.3 \mathrm{MPa} \mu \mathrm{m}$ can be interpreted as an indicator of a back stress lamella boundary resistance. The part of the flow stress caused by the cells is $G b / L \approx 350 \mathrm{MPa}$. These values correspond roughly to the measured data presented in [3].

\section{Conclusions}

- The deformation bands in the form of lamellae filled with the bamboo cells dominate the deformation microstructure at very high strains. The resistance to the dislocation flow through the laminar structure determines the maximum strength achievable by plastic deformation.

- The variational formulation shows that due to anisotropic hardening, an inhomogeneous deformation leading to the lamellae formation is energetically more favorable than the homogeneous deformation.
- Despite drastic simplification, the proposed model points to two main reasons for the formation of the laminar structure; hardening anisotropy, and dislocation cells and lamella boundaries.

\section{Acknowledgments}

This work was supported by GAČR through the project 17-04301S.

\section{References}

[1] B. Bay, N. Hansen, D.A. Hughes, D. KuhlmannWilsdorf, Acta Metall. Mater. 40, 205 (1992).

[2] D. Kuhlmann-Wilsdorf, Acta Mater. 47, 1697 (1999).

[3] D.A. Hughes, Mater. Sci. Eng. A 319-321, 46 (2001).

[4] N. Hansen, D. Juul Jensen, Mater. Sci. Technol. 27, 1229 (2011).

[5] D.A. Hughes, Acta Metall. Mater. 41, 1421 (1993).

[6] D.A. Hughes, N. Hansen, Phys. Rev. Lett. 87, 135503 (2001).

[7] D.A. Hughes, N. Hansen, Phys. Rev. Lett. 112 , 135503 (2014).

[8] M. Ortiz, E.A. Repetto, J. Mech. Phys. Solids 47, 397 (1999).

[9] M. Ortiz, E.A. Repetto, L. Stainier, J. Mech. Phys. Solids 48, 2077 (2000).

[10] H. Petryk, M. Kursa, Arch. Mech. 63, 287 (2011).

[11] H. Petryk, M. Kursa, J. Mech. Phys. Solids 61, 1854 (2013).

[12] S. Mahesh, Philos. Mag. 92, 2286 (2012).

[13] M. Arul Kumar, S. Mahesh, Int. J. Plast. 44, 95 (2013).

[14] J. Kratochvíl, M. Kružík, Philos. Mag. A 95, 3621 (2015).

[15] J. Kratochvíl, M. Kružík, Int. J. Plast. 81, 196 (2016).

[16] M.A. Biot, Mechanics of Incremental Deformations, Wiley, New York 1965.

[17] S.V. Harren, H.E. Dève, R.J. Asaro, Acta Metall. 36 2435 (1988).

[18] D.A. Hughes, Q. Liu, D.C. Chrzan, N. Hansen, Acta Mater. 45, 105 (1997). 